\title{
Elastic bending modulus of monolayer graphene
}

\author{
Qiang Lu ${ }^{1}$, Marino Arroyo ${ }^{2}$ and Rui Huang ${ }^{1}$ \\ ${ }^{1}$ Department of Aerospace Engineering and Engineering Mechanics, University of Texas, Austin, \\ TX 78712, USA \\ ${ }^{2}$ Department of Applied Mathematics 3, LaCàN, Universitat Politècnica de Catalunya (UPC), \\ Barcelona 08034, Spain
}

\begin{abstract}
An analytic formula is derived for the elastic bending modulus of monolayer graphene based on an empirical potential for solid-state carbon atoms. Two physical origins are identified for the non-vanishing bending stiffness of the atomically thin graphene sheet, one due to the bond-angle effect and the other resulting from the bond-order term associated with the dihedral angles. The analytical prediction compares closely with ab initio energy calculations. Pure bending of graphene monolayers into cylindrical tubes is simulated by a molecular mechanics approach, showing slight nonlinearity and anisotropy in the tangent bending modulus as the bending curvature increases. An intrinsic coupling between bending and in-plane strain is noted for graphene monolayers rolled into carbon nanotubes.
\end{abstract}

The unique two-dimensional (2D) lattice structure and physical properties of graphene have attracted tremendous interest recently. In particular, rippling of suspended graphene monolayers has been observed, with mesoscopic amplitude and wavelength [1]. Imaging of monolayer graphene sheets on silicon dioxide has also shown structural corrugation [2,3]. Theoretical studies [4-6] have suggested that bending stiffness of the monolayer graphene is critical in attaining the structural stability and morphology for both suspended and supported graphene sheets, which in turn could have important impacts on their electronic properties. Furthermore, single-walled carbon nanotubes are essentially graphene monolayers subjected to cylindrical bending along the particular chiral directions. It has been reported that the bending curvature (inverse of the tube radius) and bending orientation (tube chirality) have dramatic effects on both the mechanical and electronic properties of carbon nanotubes $[7,8]$. An accurate account of the bending modulus of graphene is thus important for understanding the mechanics of carbon nanotubes.

While the in-plane mechanical properties (elastic modulus and strength) of monolayer graphene have been deduced from experiments $[9,10]$, direct measurement of bending stiffness of monolayer graphene has not been reported in the literature. The often cited experimental value of $1.2 \mathrm{eV}(\sim 0.192 \mathrm{nN} \mathrm{nm})$ was derived from the phonon spectrum of graphite [11]. Theoretically, bending modulus of monolayer graphene has been predicted based on empirical potentials [7, 12-14] and $a b$ initio calculations $[15,16]$. The fact that the atomically thin graphene monolayer has a finite bending modulus is in contrast to classical theories for plates and shells [17]. For example, the bending modulus of an elastic thin plate scales with the cube of its thickness, namely,

$$
D=\frac{\mathrm{d} M}{\mathrm{~d} \kappa} \sim E h^{3}
$$

where $M$ is the bending moment, $\kappa$ the bending curvature, $h$ the plate thickness and $E$ Young's modulus. The linear relationship between the bending modulus and Young's modulus in equation (1) is a result of the classical Kirchhoff hypothesis [17], which assumes linear variation of the strain and stress along the thickness of a thin elastic plate. For a graphene monolayer, however, its physical thickness cannot 


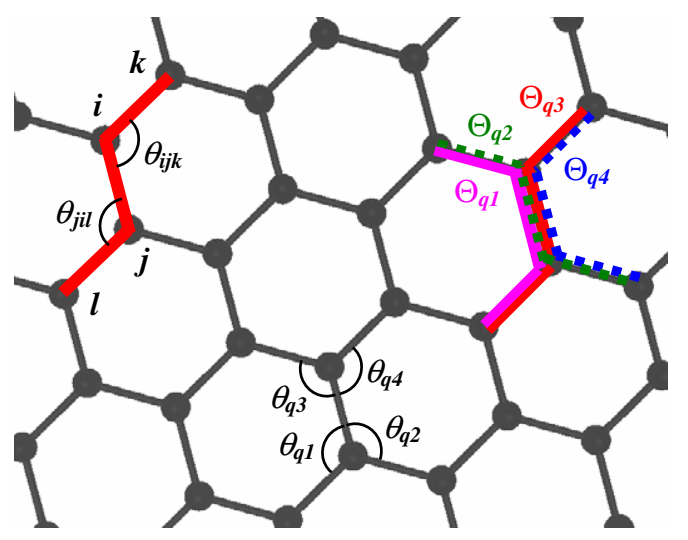

Figure 1. (Colour online.) Illustration of bond angles and dihedral angles in graphene lattice. Each dihedral angle is represented by three bonds connecting four carbon atoms (e.g. $i, j, k, l$ ), and each carbon-carbon bond is associated with four bond angles and four dihedral angles.

be defined unambiguously in the continuum sense [14], and the Kirchhoff hypothesis simply does not apply. Therefore, different physical origins must be sought for the bending moment and bending modulus in graphene monolayers.

Based on the first-generation Brenner potential [18], a simple analytical form was derived for the bending modulus of monolayer graphene under infinitesimal bending curvature $[13,14]$, which established a clear connection between the functional form of the empirical potential and the effective bending modulus. The potential takes the form

$$
V_{i j}=V_{R}\left(r_{i j}\right)-\bar{b}_{i j} V_{A}\left(r_{i j}\right),
$$

where $i$ and $j$ are indices labelling the atoms, $r_{i j}$ is the bond length and $\bar{b}_{i j}=\left(b_{i j}+b_{j i}\right) / 2$ is the bond-order function describing the bonding environment. Near the ground state of graphene, a cutoff function in the potential restricts the repulsive and attractive interaction functions, $V_{\mathrm{R}}(r)$ and $V_{\mathrm{A}}(r)$, to the nearest neighbours, while the bond-order function $\bar{b}_{i j}$ accounts for many-body interactions up to the second nearest neighbours through its dependence on the bond angles, $\theta_{i j k}$ and $\theta_{j i k}(k \neq i, j)$, as illustrated in figure 1. As shown in $[13,14]$, at the ground state of a planar graphene monolayer, with $r_{i j}=r_{0}$ and all the bond angles identically $2 \pi / 3$, the bending modulus derived from the empirical potential is

$$
D=\frac{\sqrt{3}}{2}\left(\frac{\partial V_{i j}}{\partial \cos \theta_{i j k}}\right)_{g}=\frac{1}{2} V_{A}\left(r_{0}\right) b^{\prime}(2 \pi / 3,2 \pi / 3),
$$

where the subscript $g$ denotes derivatives at the ground state and $b^{\prime}$ denotes the derivative of the function $b_{i j}$ with respect to either one of the two bond angles. Equation (3) reveals that the physical origin of the bending modulus comes from multibody interactions of the carbon atoms through the bondangle effect in the interatomic potential. In other words, any empirical potential with only the nearest neighbour (twobody) interactions would lead to zero bending modulus of the monolayer.

Using the second set of the parameters for the Brenner potential [18], the bending modulus predicted by equation (3) is $D=0.133 \mathrm{nN} \mathrm{nm}$, or equivalently, $0.83 \mathrm{eV}$. This prediction, however, is considerably lower than that from ab initio energy calculations with $D=3.9 \mathrm{eV} \AA^{2} /$ atom [16], or equivalently, $0.238 \mathrm{nN} \mathrm{nm}(1.5 \mathrm{eV})$. Applying the same equation for the second-generation Brenner potential [19] leads to an even lower bending modulus: $D=0.110 \mathrm{nNnm}$ or $0.69 \mathrm{eV}$. The discrepancy between the analytical prediction of equation (3) and the $a b$ initio calculations suggests that the bond-angle effect in the empirical potentials does not fully account for the bending stiffness of the monolayer graphene. In this paper we resolve this discrepancy by showing that, in addition to the bond-angle effect, the dihedral angle effect must be included in the consideration of bending energetics of graphene. With many-body interactions up to the third nearest neighbours, the dihedral angle effect adds a significant contribution to the bending stiffness of monolayer graphene and thus the bending energy in carbon nanotubes.

While several empirical potentials (including Tersoff [12] and first-generation Brenner potentials [18]) consider multibody interactions up to the second nearest neighbours, the second-generation Brenner potential for carbon includes the third nearest neighbours via a bond-order term associated with the dihedral angles [19]. Taking the same general form as equation (2), the bond-order function for the second-generation Brenner potential is

$$
\bar{b}_{i j}=\frac{1}{2}\left(b_{i j}^{\sigma-\pi}+b_{j i}^{\sigma-\pi}\right)+b_{i j}^{\mathrm{DH}}+\Pi_{i j}^{\mathrm{RC}},
$$

where $\frac{1}{2}\left(b_{i j}^{\sigma-\pi}+b_{j i}^{\sigma-\pi}\right)$ is a function of the bond angles similar to that in the first-generation Brenner potential, $b_{i j}^{\mathrm{DH}}$ is a function of the dihedral angles and $\Pi_{i j}^{\mathrm{RC}}$ represents the influence of radical energetics and $\pi$-bond conjugation on the bond energy. For a perfect graphene lattice with no vacancy, $\Pi_{i j}^{\mathrm{RC}}=0$, and the dihedral function takes the form

$$
b_{i j}^{\mathrm{DH}}=\frac{T_{0}}{2} \sum_{k, l(\neq i, j)}\left[\left(1-\cos ^{2} \Theta_{i j k l}\right) f_{\mathrm{c}}\left(r_{i k}\right) f_{\mathrm{c}}\left(r_{j l}\right)\right],
$$

where $T_{0}=-0.00809675$ and $f_{\mathrm{c}}(r)$ is the cutoff function that restricts the dihedral function to the four nearest neighbours ( $k$ and $l$ ) of the atoms $i$ and $j$, as illustrated in figure 1 . The dihedral angle can be determined by

$$
\cos \Theta_{i j k l}=\boldsymbol{n}_{j i k} \cdot \boldsymbol{n}_{i j l}
$$

where $\boldsymbol{n}_{j i k}$ and $\boldsymbol{n}_{i j l}$ are the unit normal vectors to the planes of the triangles $j i k$ and $i j l$, respectively, namely,

$$
\boldsymbol{n}_{j i k}=\frac{\boldsymbol{r}_{j i} \times \boldsymbol{r}_{i k}}{r_{i j} r_{i k} \sin \theta_{i j k}} \quad \text { and } \quad \mathrm{n}_{i j l}=\frac{\boldsymbol{r}_{i j} \times \boldsymbol{r}_{j l}}{r_{i j} r_{j l} \sin \theta_{j i l}} .
$$

Therefore, each $\mathrm{C}-\mathrm{C}$ bond in the graphene lattice is associated with four dihedral angles, and each dihedral angle accounts for an interaction between one atom (e.g. atom $k$ ) and one of its third nearest neighbours (e.g. atom $l$ ). For a planar graphene monolayer, the dihedral angles are either 0 or $\pi$, and thus $b_{i j}^{\mathrm{DH}}=0$. However, the dihedral term becomes non-zero upon bending of the graphene monolayer. 
Following a similar approach as in [13], the bending modulus of monolayer graphene is derived as follows:

$$
D=\frac{\partial^{2} W}{\partial \kappa^{2}}
$$

where $W$ is the strain energy density function depending on the bond lengths, bond angles, and dihedral angles in a unit cell of graphene. Each unit cell contains two carbon atoms and three inequivalent bonds (relabelled as $q=1-3$ ), and the strain energy density can be obtained by summing up the interatomic potential energy, namely,

$$
\begin{aligned}
W= & \frac{1}{S_{0}} \sum_{q=1}^{3}\left[V_{\mathrm{R}}\left(r_{q}\right)-\bar{b}\left(\theta_{q 1}, \ldots, \theta_{q 4}, \Theta_{q 1}, \ldots, \Theta_{q 4}\right)\right. \\
& \left.\times V_{\mathrm{A}}\left(r_{q}\right)-V_{0}\right],
\end{aligned}
$$

where $S_{0}=\frac{3}{2} \sqrt{3} r_{0}^{2}$ is the area of the unit cell at the ground state, $V_{0}$ is the ground-state bond energy and the bond-order function has been written explicitly in terms of the four bond angles and four dihedral angles for each bond (figure 1). In the following derivation, it must be checked that the internal lattice relaxation within the unit cell does not affect the bending modulus, as it was done in [13] in the absence of the dihedral terms. This has been confirmed to hold in the present setting as well.

At the ground state of graphene, it can be shown that

$$
\begin{aligned}
& \left(\frac{\partial r_{q}}{\partial \kappa}\right)_{g}=0, \quad\left(\frac{\partial \theta_{q k}}{\partial \kappa}\right)_{g}=0 \quad \text { and } \\
& \left(\frac{\partial \cos \Theta_{q k}}{\partial \kappa}\right)_{g}=0 .
\end{aligned}
$$

Therefore, the bending modulus of graphene under an infinitesimal bending curvature from the ground state is

$$
\begin{aligned}
D= & \sum_{q=1}^{3} \frac{\partial W}{\partial r_{q}} \frac{\partial^{2} r_{q}}{\partial \kappa^{2}}+\sum_{q=1}^{3} \sum_{k=1}^{4} \frac{\partial W}{\partial \theta_{q k}} \frac{\partial^{2} \theta_{q k}}{\partial \kappa^{2}} \\
& +\sum_{q=1}^{3} \sum_{k=1}^{4} \frac{\partial W}{\partial \cos \Theta_{q k}} \frac{\partial^{2} \cos \Theta_{q k}}{\partial \kappa^{2}} .
\end{aligned}
$$

Furthermore, taking derivatives of the strain energy at the ground state, we have

$$
\begin{aligned}
& \left(\frac{\partial W}{\partial r_{q}}\right)_{g}=0, \quad\left(\frac{\partial W}{\partial \theta_{q k}}\right)_{g}=-\frac{V_{\mathrm{A}}\left(r_{0}\right)}{2 S_{0}}\left(b_{0}^{\sigma-\pi}\right)^{\prime}, \\
& \left(\frac{\partial W}{\partial \cos \Theta_{q k}}\right)_{g}= \pm \frac{T_{0}}{S_{0}} V_{\mathrm{A}}\left(r_{0}\right) .
\end{aligned}
$$

where $\left(b_{0}^{\sigma-\pi}\right)^{\prime}$ denotes the derivative of the function $b_{i j}^{\sigma-\pi}$ with respect to either one of the two bond angles at the ground state. Thus, equation (11) becomes

$$
\begin{aligned}
D= & -\frac{V_{\mathrm{A}}\left(r_{0}\right)}{2 S_{0}}\left(b_{0}^{\sigma-\pi}\right)^{\prime} \sum_{q=1}^{3} \sum_{k=1}^{4}\left(\frac{\partial^{2} \theta_{q k}}{\partial \kappa^{2}}\right)_{g} \\
& +\frac{T_{0} V_{\mathrm{A}}\left(r_{0}\right)}{S_{0}} \sum_{q=1}^{3} \sum_{k=1}^{4}\left(\cos \Theta_{q k} \frac{\partial^{2} \cos \Theta_{q k}}{\partial \kappa^{2}}\right)_{g} .
\end{aligned}
$$

As shown in [13], $\sum_{q=1}^{3} \sum_{k=1}^{4}\left(\partial^{2} \theta_{q k} / \partial \kappa^{2}\right)_{g}=-9 / 2 \sqrt{3} r_{0}^{2}$. Using a method of asymptotic expansion, we find that

$$
\sum_{q=1}^{3} \sum_{k=1}^{4}\left(\cos \Theta_{q k} \frac{\partial^{2} \cos \Theta_{q k}}{\partial \kappa^{2}}\right)_{g}=-\frac{21 r_{0}^{2}}{2} .
$$

Finally, we obtain a new analytical form for the bending modulus of monolayer graphene:

$$
D=\frac{V_{\mathrm{A}}\left(r_{0}\right)}{2}\left(\left(b_{0}^{\sigma-\pi}\right)^{\prime}-\frac{14 T_{0}}{\sqrt{3}}\right) \text {. }
$$

While the first term on the right-hand side of equation (15) is identical to equation (3), the second term results from the effect of dihedral angles in the second-generation Brenner potential. With the additional term, equation (15) predicts that $D=0.225 \mathrm{nN} \mathrm{nm}(1.4 \mathrm{eV})$, very close to the prediction from the $a b$ initio calculations [16]. It is thus suggested that the effect of dihedral angles plays an important role in bending of graphene monolayers. This formula clearly shows how the multibody interactions, including both the second and third nearest neighbours, contribute to the finite bending stiffness of the atomically thin membrane governed by a bond-order potential. While the specific potential is used in the above derivation, the procedure and result can be readily generalized for other empirical potentials.

To validate the analytical expression for the bending modulus of monolayer graphene, we carry out atomistic simulations in which graphene monolayers are rolled into cylindrical tubes of various diameters. The static molecular mechanics (MM) approach is adopted to calculate the strain energy, from which the bending moment and bending modulus are deduced. In previous studies [12, 13, 16], strain energies of fully relaxed carbon nanotubes were calculated as a function of the curvature $(\kappa=1 / R)$. It has been noted that, relative to the ground state of planar graphene, the deformation of a fully relaxed carbon nanotube involves both the bending curvature and in-plane strain [20,21]. Consequently, the strain energy of a fully relaxed carbon nanotubes should include contributions from in-plane strain and thus cannot be written simply as a quadratic function of the curvature. To achieve pure bending (with zero in-plane strain) of the graphene monolayers, in our simulations the total potential energy is minimized under the constraint that the tube radius and length do not change. The constraint on the tube length is easily applied by the periodic boundary condition along the axial direction of the tube. To enforce the constraint on the tube radius, the graphene is first rolled up by mapping a 2D sheet of width $a$ onto a cylindrical tube of radius $R=a / 2 \pi$. Next, the potential energy is minimized by internal relaxation between the two sublattices of graphene, with one sublattice held in place and the other allowed to relax. In this way, the overall tube radius does not change during the energy minimization step. The resulting tubes from these simulations are not fully relaxed; in other words, external reaction forces are required to keep the tube dimensions from relaxing, which include forces in both the 


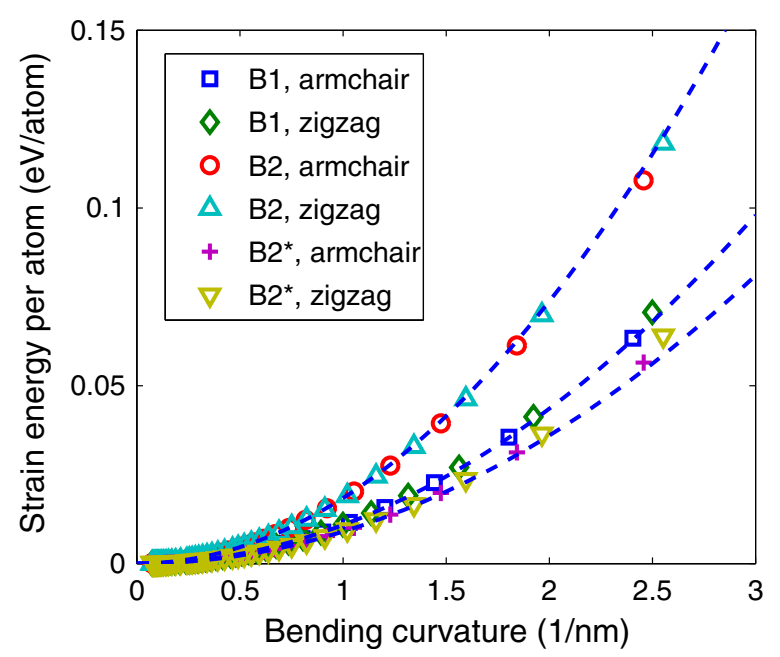

Figure 2. (Colour online.) Strain energy per atom in graphene tubes as a function of bending curvature obtained from different empirical potentials: B1 for the first-generation Brenner potential, B2 for the second-generation Brenner potential and $\mathrm{B} 2 *$ for the

second-generation Brenner potential without considering the dihedral term. Results from molecular mechanics simulations are shown for pure bending of monolayer graphene along the armchair and zigzag directions, while the quadratic function, $W=D \kappa^{2} / 2$, is plotted as the dashed line using the analytical bending modulus for each potential.

axial and radial directions. With the pure bending deformation, the strain energy density of the constrained tube depends only on the tube radius or curvature. In the linear elastic regime, we have $W=\frac{1}{2} D \kappa^{2}$, and the bending moment is simply, $M=\mathrm{d} W / \mathrm{d} \kappa=D \kappa$, with the bending modulus $D$ as given in equation (15).

Figure 2 plots the strain energy per atom as a function of the curvature for graphene monolayers rolled along the armchair and zigzag directions. For comparison, the results from both the first and second-generation Brenner potentials (B1 and B2) are shown. To further highlight the effect of dihedral angles, also shown are the results from simulations ignoring the dihedral term in the second-generation Brenner potential $\left(\mathrm{B} 2^{*}\right)$. Clearly, the strain energy for potential B2 is systematically higher than the other two. The dihedral term contributes significantly to the bending energy, especially for large bending curvatures (small nanotubes). The corresponding bending moments are obtained by numerically differentiating the strain energy with respect to the bending curvature, as plotted in figure 3 . In all three cases, the bending moment increases almost linearly with the curvature up to $2 \mathrm{~nm}^{-1}$, with slight nonlinearity at large curvatures. By further differentiating the bending moment with respect to the curvature, we obtain the tangent bending modulus, as plotted in figure 4 . The tangent modulus at small curvatures agrees closely with the analytical prediction by equation (15) as indicted by the dashed lines for the three potentials. At large curvatures (carbon nanotubes of small radii), the tangent bending modulus deviates slightly as a result of nonlinearity. Due to the effect of dihedral angles, the tangent modulus obtained from the potential B2 is considerably higher than those from B1 and B2*. Interestingly, the tangent bending

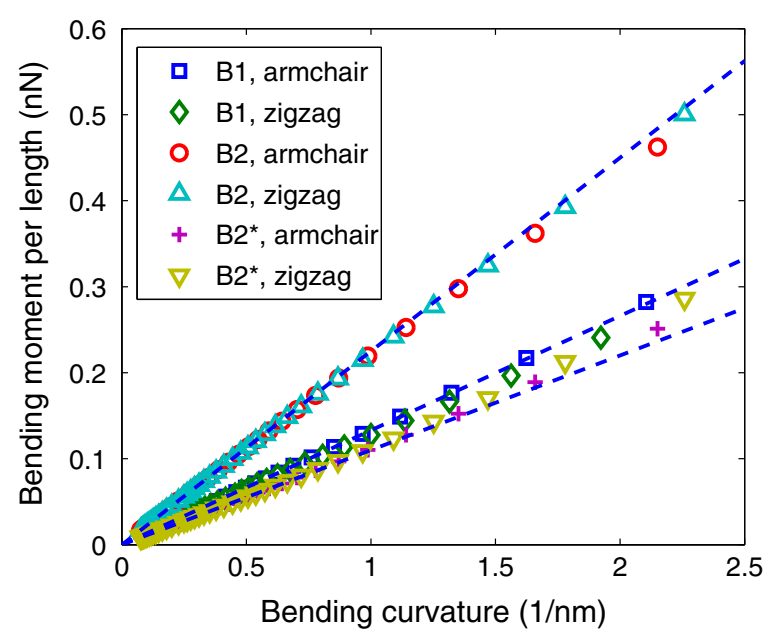

Figure 3. (Colour online.) Bending moment in graphene tubes versus bending curvature along the armchair and zigzag directions, obtained from different empirical potentials. The linear elastic bending moment-curvature relation, $M=D \kappa$, is plotted as the dashed line using the analytical bending modulus for each potential.

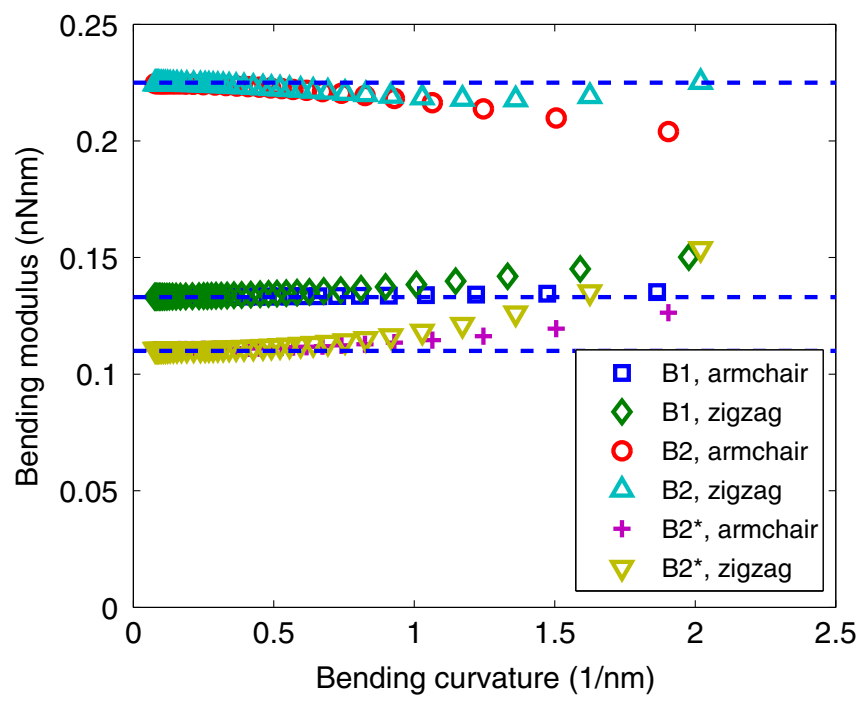

Figure 4. (Colour online.) Tangent bending modulus of monolayer graphene as a function of bending curvature along the armchair and zigzag directions, obtained from different empirical potentials. The analytical prediction for the linear elastic bending modulus (independent of curvature) is plotted as the dashed line for each potential.

modulus from B2 decreases slightly as the curvature increases, while the trend is reversed when the dihedral term is ignored in potentials $\mathrm{B} 2 *$ and $\mathrm{B} 1$.

Figure 4 shows that the tangent bending moduli along the zigzag and armchair directions are essentially identical at small curvatures, but become increasingly different as the curvature increases. As expected, the monolayer graphene at the ground state is elastically isotropic due to the hexagonal symmetry of the graphene lattice, and the bending modulus at the linear elastic regime as predicted by equation (15) is independent of the bending direction. However, the lattice symmetry is distorted by the cylindrical bending deformation and the monolayer graphene becomes slightly anisotropic at 


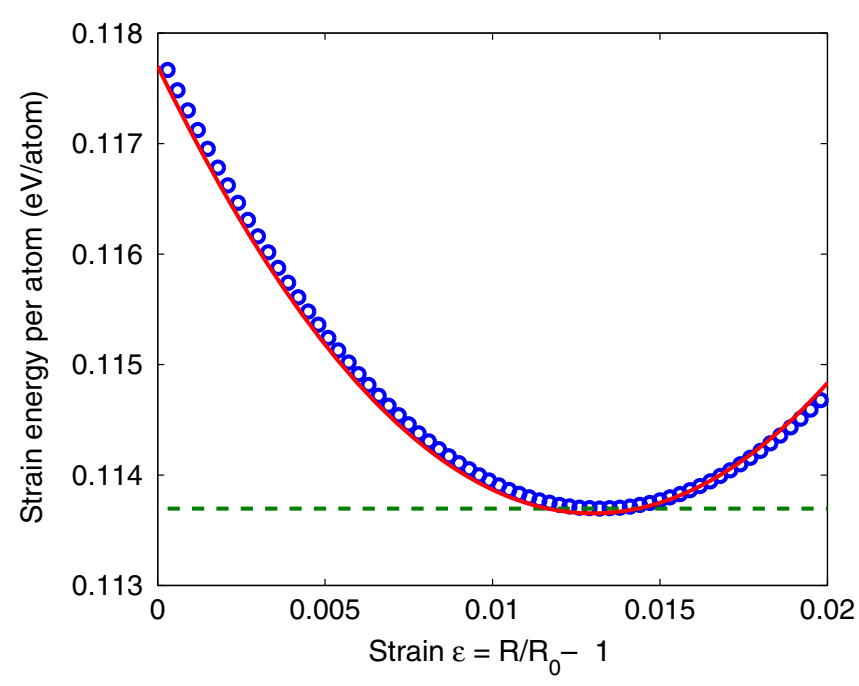

Figure 5. (Colour online.) Relaxation of the strain energy for a $(10,0)$ carbon nanotube as its radius increases. The atomistic calculations are shown by open circles and the prediction by equation (16) is plotted as the solid curve. The dashed line indicates the strain energy from an atomistic simulation without imposing any constraint on the tube radius.

the nonlinear regime. Even stronger anisotropy was predicted for the in-plane elastic moduli of monolayer graphene under finite stretches [20].

It is noted that, due to the constraint on the tube radius and length, the strain energy of pure bending (figure 2) is slightly higher than the corresponding strain energy in fully relaxed carbon nanotubes [13]. To illustrate the effect of the constraint, figure 5 plots the strain energy as a function of the tube radius for a $(10,0)$ carbon nanotube. The tube radius $R$ is gradually increased in the MM simulations, while the tube length remains fixed. Only the second-generation Brenner potential with the dihedral term is used here. As shown in figure 5, the strain energy decreases until it reaches a minimum at $R / R_{0} \sim 1.013$, where $R_{0}=0.397 \mathrm{~nm}$ is the tube radius before relaxation. The minimum energy is a few percent lower than the pure bending energy and it compares closely with the corresponding strain energy by MM calculations without imposing any constraint on the tube radius. Therefore, relaxation of the radial constraint alone leads to increase in the tube radius by about $1.3 \%$ or an in-plane strain $\varepsilon=0.013$ in the circumferential direction of the tube. Further relaxation in the axial direction would result in even lower strain energy along with slightly different tube radius and length for a fully relaxed nanotube [21].

To understand the energy reduction and radius increase in the relaxed tube, one may consider the total strain energy as the sum of the bending energy and the in-plane membrane strain energy. As the tube radius increases, the bending energy, $W_{\mathrm{B}}=D \kappa^{2} / 2=D /\left(2 R^{2}\right)$, decreases and the membrane strain energy, $W_{\mathrm{M}}=C \varepsilon^{2} / 2$, increases, where $D$ and $C$ are the elastic modulus for bending and in-plane stretch, respectively. However, with $D=0.225 \mathrm{nN} \mathrm{nm}$ and $C=289 \mathrm{~N} \mathrm{~m}^{-1}$ for the second-generation Brenner potential $[13,20]$, the competition between the two energy terms leads to a minimum strain energy at $\varepsilon \sim 0.005$ for the $(10,0)$ nanotube, much smaller than that in figure 5. More accurately, we expand the strain energy with respect to the bending curvature and membrane strain to the leading orders, namely,

$W(\kappa, \varepsilon) \approx \frac{1}{2} D \kappa_{0}^{2}+M\left(\kappa-\kappa_{0}\right)+\sigma \varepsilon+\frac{1}{2} D\left(\kappa-\kappa_{0}\right)^{2}+\frac{1}{2} C \varepsilon^{2}$,

where $\kappa_{0}=1 / R_{0}$ is the curvature before relaxation, $M=$ $(\partial W / \partial \kappa)_{\varepsilon=0}$ is the bending moment and $\sigma=(\partial W / \partial \varepsilon)_{\varepsilon=0}$ is the in-plane membrane force in the circumferential direction for the un-relaxed tube. The strain energy then has a minimum at $\varepsilon=\left(D \kappa_{0}^{2}-\sigma\right) /\left(C+3 D \kappa_{0}^{2}\right)$. For the $(10,0)$ nanotube, we find that, with $\sigma=-2.38 \mathrm{~N} \mathrm{~m}^{-1}$, equation (16) agrees closely with the $\mathrm{MM}$ calculations in figure 5 for the in-plane strain up to a few percent. Therefore, the tube is subject to a compressive membrane force along the circumferential direction before relaxation. The compressive membrane force may be qualitatively understood as a result of shortening of the bond lengths in the constrained tube, relative to the bond length at the ground state of graphene. The imposed constraint over the tube radius effectively applies an external pressure onto the tube, balancing the internal membrane force. The applied external pressure may be estimated from the Laplace-Young equation, namely, $p=\sigma / R_{0}=6.0 \mathrm{GPa}$, which compares closely with $a b$ initio calculations of carbon nanotubes under hydrostatic pressure [22]. We note that the presence of the membrane force before relaxation is in clear contrast with the classical plate theory which predicts zero membrane force under the pure bending condition. This suggests an intrinsic coupling between bending and in-plane strain due to the discrete nature of the graphene lattice.

In summary, we have shown that the non-vanishing bending modulus of the atomically thin graphene monolayers results from multibody atomistic interactions up to the third nearest neighbours, as accounted for in a bond-order empirical potential by the effects of bond angles (second nearest neighbours) and the dihedral angles (third nearest neighbours). A new analytical expression is derived for the elastic bending modulus at the ground state, which compares closely with $a b$ initio calculations. Slight nonlinearity and anisotropy are noted for tangent bending modulus obtained from molecular mechanics based atomistic simulations. An intrinsic coupling between bending and in-plane strain is suggested, which leads to a compressive membrane force under pure bending and reduction in the strain energy in relaxed carbon nanotubes.

\section{Acknowledgments}

The authors (RH and QL) gratefully acknowledge funding of this work by the US Department of Energy through Grant No DE-FG02-05ER46230. MA acknowledges the support received through the grant DPI2007-61054 of the Ministerio de Ciencia e Innovacion and through the prize 'ICREA Academia' for excellence in research, funded by the Generalitat de Catalunya.

\section{References}

[1] Meyer J C, Geim A K, Katsnelson M I, Novoselov K S, Booth T J and Roth S 2007 The structure of suspended graphene sheets Nature 446 60-3 
[2] Ishigami M, Chen J H, Cullen W G, Fuhrer M S and Williams E D 2007 Atomic structure of graphene on $\mathrm{SiO}_{2}$ Nano Lett. 7 1643-8

[3] Stolyarova E, Rim K T, Ryu S, Maultzsch J, Kim P, Brus L E, Heinz T F, Hybertsen M S and Flynn G W 2007

High-resolution scanning tunneling microscopy imaging of mesoscopic graphene sheets on an insulating surface Proc. Natl Acad. Sci. USA 104 9209-12

[4] Fasolino A, Los J H and Katsnelson M I 2007 Intrinsic ripples in graphene Nature Mater. 6 858-61

[5] Abedpour N et al 2007 Roughness of undoped graphene and its short-range induced gauge field Phys. Rev. B 76195407

[6] Kim E-A and Neto A H C 2008 Graphene as an electronic membrane Eur. Phys. Lett. 8457007

[7] Tu Z-C and Ou-Yang Z-C 2002 Single-walled and multiwalled carbon nanotubes viewed as elastic tubes with the effective Young's modulus dependent on layer number Phys. Rev. B 65233407

[8] Saito R, Dresselhaus R G and Dresselhaus M S 1998 Physical Properties of Carbon Nanotubes (London: Imperial College Press)

[9] Lee C, Wei X D, Kysar J W and Hone J 2008 Measurement of the elastic properties and intrinsic strength of monolayer graphene Science $321385-8$

[10] Bunch J S, van der Zande A M, Verbridge S S, Frank I W, Tanenbaum D M, Parpia J M, Craighead H G and McEuen P L 2007 Electromechanical resonators from graphene sheets Science 315 490-3

[11] Nicklow R, Wakabayashi N, and Smith H G 1972 Lattice dynamics of pyrolytic graphite Phys. Rev. B 5 4951-62
[12] Tersoff J 1988 New empirical approach for the structure and energy of covalent systems Phys. Rev. B 37 6991-7000

[13] Arroyo M and Belytschko T 2004 Finite crystal elasticity of carbon nanotubes based on the exponential Cauchy-Born rule Phys. Rev. B 69115415

[14] Huang Y, Wu J and Hwang K C 2006 Thickness of graphene and single-wall carbon nanotubes Phys. Rev. B 74033524

[15] Sanchez-Portal D, Artacho E, Soler J M, Rubio A and Ordejon P 1999 Ab initio structural, elastic, and vibrational properties of carbon nanotubes Phys. Rev. B 59 12678-88

[16] Kudin K N, Scuseria G E and Yakobson B I 2001 C2F, BN, and $\mathrm{C}$ nanoshell elasticity from ab initio computations Phys. Rev. B 64235406

[17] Timoshenko S and Woinowsky-Krieger S 1987 Theory of Plates and Shells (New York: McGraw-Hill)

[18] Brenner D W 1990 Empirical potential for hydrocarbons for use in simulating the chemical vapor deposition of diamond films Phys. Rev. B 42 9485-71

[19] Brenner D W, Shenderova O A, Harrison J A, Stuart S J, Ni B and Sinnott S B 2002 A second-generation reactive empirical bond order (REBO) potential energy expression for hydrocarbons J. Phys.: Condens. Matter 14 783-802

[20] Lu Q and Huang R 2009 Nonlinear mechanics of single-atomic-layer graphene sheets J. Comput. Theor. Nanosci. at press

[21] Wu J, Hwang K C and Huang Y 2008 An atomistic-based finite deformation shell theory for single-wall carbon nanotubes J. Mech. Phys. Solids 56 279-92

[22] Reich S, Thomsen C and Ordejon P 2002 Elastic properties of carbon nanotubes under hydrostatic pressure Phys. Rev. B $\mathbf{6 5} 153407$ 